\title{
Correction to: Synthesis and Characterization of Plasma-Polymer Gate Dielectric Films for Graphene Field Effect Transistor Devices
}

\author{
Hyeon Jin Seo ${ }^{1,2} \cdot$ Yeong Eun Gil ${ }^{1} \cdot \mathrm{Ki}-H w a n \mathrm{Hwang}^{2} \cdot$ Antony Ananth $^{1,2} \cdot$ Jin-Hyo Boo ${ }^{1,2}$ (i)
}

Published online: 12 June 2019

(c) The Korean Institute of Metals and Materials 2019

\section{Correction to: \\ Electronic Materials Letters (2019) 15:396-401 \\ https://doi.org/10.1007/s13391-019-00139-6}

The original version of this article unfortunately contains a mistake. Two numbers in the acknowledgement section are incorrect. The correct acknowledgements are as follows:

Acknowledgements This work was supported by a grant from the National Research Foundation of Korea (NRF) funded by the Korean government (NRF-2018R1D1A1B07051012 and NRF-2017R1D1A1B03029848).
Publisher's Note Springer Nature remains neutral with regard to jurisdictional claims in published maps and institutional affiliations.

The original article can be found online at https://doi.org/10.1007/ s13391-019-00139-6.

Jin-Hyo Boo

jhboo@skku.edu

1 Department of Chemistry, Sungkyunkwan University, Suwon 16419, Republic of Korea

2 Institute of Basic Science, Sungkyunkwan University, Suwon 16419, Republic of Korea 